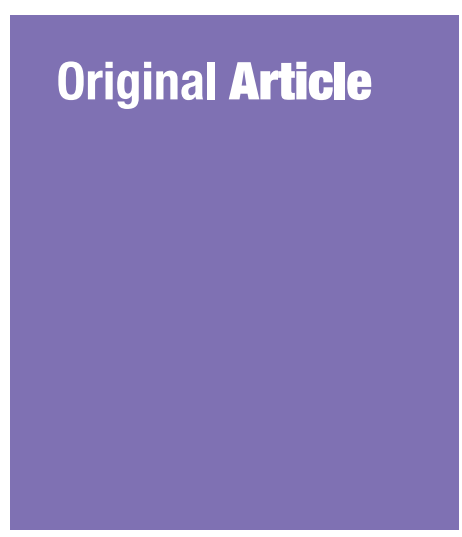

Submitted: 9 Jan 2016 Accepted: 2 Aug 2016 Online: 24 Feb 2017

\section{Prevalence of Shunt Dependency and Clinical Outcome in Patients with Massive Intraventricular Haemorrhage Treated with Endoscopic Washout and External Ventricular Drainage}

\author{
Jason Raj Johnson ${ }^{3}$, ZamzURI Idris ${ }^{1,2}$, JafRI Malin Abdullah ${ }^{1,2}$, \\ Azmı Alias $^{3}$, Mohammad SAfFarı Mohammad Haspani ${ }^{3}$
}

Department of Neurosciences, School of Medical Sciences, Universiti Sains Malaysia, 16150 Kubang Kerian, Kelantan, Malaysia

2 Center for Neuroscience Service and Research, School of Medical Sciences, Universiti Sains Malaysia, 16150 Kubang Kerian, Kelantan, Malaysia

3 Department of Neurosurgery, Kuala Lumpur General Hospital, 50586, Kuala Lumpur, Malaysia

To cite this article: Johnson JR, Zamzuri I, Jafri Malin A, Azmi A, Mohammad Saffari MH. Prevalence of shunt dependency and clinical outcome in patients with massive intraventricular haemorrhage treated with endoscopic washout and external ventricular drainage. Malays $J$ Med Sci. 2017;24(1):40-46. https://doi.org/10.21315/ mjms2017.24.1.5

To link to this article: https://doi.org/10.21315/mjms2017.24.1.5

\title{
Abstract
}

Background: Intraventricular haemorrhage (IVH) causes blockage of ventricular conduits leading to hydrocephalus, increased intracranial pressure (ICP), and a reduced level of consciousness. The current standard management of IVH is insertion of an external ventricular drainage (EVD) catheter. However, this procedure addresses only the problems of acute hydrocephalus and raised ICP. Endoscopic washout allows for a more complete removal of the intraventricular clot. This study compared these two types of treatment in terms of shunt dependency and relevant clinical outcomes.

Methods: Patients who were 10-80 years old and presented with a Graeb score of more than six were randomised into endoscopic washout and EVD treatment groups. A CT brain was repeated on each patient within 24 hours after surgery, and if a patient's Graeb score was still more than six, a repeat endoscopic washout was performed to clear the remaining clots. All patients were monitored for shunt dependency at two weeks and three months, and clinical outcomes were measured at six months after the procedure.

Results: A total of 39 patients were recruited; 19 patients were randomised into the endoscopic washout group, and 20 were randomised into the EVD group. However, three patients in the endoscopic group refused that treatment and opted for EVD insertion. Patients treated with endoscopic washout had significantly less drainage dependency at two weeks $(P<0.005)$ and at three months $(P<0.004)$ as compared to patients in the external ventricular drainage group. The reduction in Graeb scores was also significantly greater in the endoscopic washout group $(P<0.001)$. However, the functional outcome at six months measured via a modified Rankin scale score was no different in the two groups of patients. The difference in the functional outcome of the patients was mainly dependent on the initial pathology, with those presenting with a thalamic bleed with IVH showing a poor functional outcome. This parameter was also influenced by the Glasgow Coma Scale (GCS) score on admission, with those patients with a score of 12 or less having a poor functional outcome (MRS 5-6) at three and six months after the surgery.

Conclusions: The use of neuroendoscopy in patients with a massive IVH significantly reduced drainage dependency. However, it did not alter the final functional outcome. 
Original Article | Endoscopy in massive intraventricular haemorrhage

\section{Introduction}

Intraventricular haemorrhage (IVH) is a devastating neurosurgical entity commonly seen in cases of hypertensive intracerebral haemorrhage (ICH) with extension into the ventricular system. It contributes to morbidity in three main ways (1). First, when a haemorrhage occurs in the ventricular system, it will organise to form clots that will block the narrow parts of the ventricular conduits, producing acute hydrocephalus. If left untreated, acute hydrocephalus can elevate intracranial pressure and lead to death. Second, the prolonged presence of a clot deep within the brain is related to a reduced level of consciousness and to mortality. EVD does not consistently improve the outcome for patients in either event. This is because drainage alone does not alter the size of the ventricles, brain tissue edema, and the inflammation provoked by the presence of intraventricular blood. Neither does it increase the rate of blood clot resolution. To worsen the situation, placement of a drain is frequently complicated by infections such as meningitis and ventriculitis, and it may also contribute to the formation of a new haemorrhage (2). Finally, blood degradation products flow via the cerebrospinal fluid (CSF) pathways to the arachnoid granulations, where prolonged contact may lead to an inflammatory response that permanently scars the granulations and causes delayed communicating hydrocephalus. Neuroendoscopy could provide a complete solution to all three clinical sequalae of IVH. Therefore, the objective of this study was to compare these two methods in terms of shunt dependency and functional clinical outcomes.

\section{Materials and Methods}

\section{Patient selection, randomisation, and exclusion criteria}

Patients who were 10-80 years old with an IVH Graeb score of 6 or more were randomised to be managed either via an endoscopic washout or an external ventricular drain from January 2010 to January 2014. Details regarding the study protocol, randomisation, and surgical procedure were explained to close family members or next of kin. Once they fully understood the study, informed consent was obtained. Institutional review board approval was also obtained (IRB Reg No: ooo04494).
Randomisation was accomplished using sealed envelopes containing the type of treatment. The envelopes were initially blinded to the consenting individuals and the treating surgeon. No blinding was done after the patients were assigned to the interventions. Those who presented with a posturing motor score, brainstem bleed, and/or the presence of coagulopathy were excluded from the study.

\section{Surgery}

In the endoscopic washout group, the procedure was done with the patient in a supine position under general anesthesia. For patients who presented with blood in both lateral ventricles, a bilateral precoronal burr hole was made. A $2.5 \mathrm{~cm}-3 \mathrm{~cm}$ long skin incision in a dural opening was made in a cruciate or inverted "U" fashion. A rigid endoscope of 6.1 $\mathrm{mm}\left(30^{\circ}\right)$ with a wide-angle, straightforward telescope was inserted into the lateral ventricle, and clot evacuation was done through the middle working channel. Warm Hartman's solution $\left(37.8^{\circ} \mathrm{C}-38.0^{\circ} \mathrm{C}\right)$ was used in irrigation until the intraventricular structures were fairly visible, and the same steps were repeated for the contralateral lateral ventricle and the third ventricle. During washout, an EVD was placed on the contralateral side to monitor ICP; this guided us to regulate the flow of the washout solution. When a clot was adherent to the ventricular wall, a size 8 Ryle's tube was introduced through the middle working channel, and gentle suction was applied until the clot detached. In patients with a clot in the cerebral aqueduct or $4^{\text {th }}$ ventricle, an endoscopic third ventriculostomy (ETV) was performed. Once the endoscopic procedure was completed, an external ventricular drain was placed. In the external ventricular drainage group, a traditional EVD insertion was performed. An incision measuring $2.5 \mathrm{~cm}-3 \mathrm{~cm}$ was made at the Kocher's point on the side with the least amount of blood in the lateral ventricle. In both groups, the EVD was placed at an initial height of $10 \mathrm{~cm}$ from the tragus.

\section{Post-operative protocol}

A repeat CT brain was done within 24 hours after the surgery, and if the Graeb score was more than six, a repeat endoscopic washout was performed to clear the remaining clots. If the drainage of cerebrospinal fluid was less than $200 \mathrm{ml} /$ day, the EVD was increased at a height of $5 \mathrm{~cm}$ daily. However, if the drainage was more than $200 \mathrm{mls} /$ day, the EVD was maintained at its previous height. If a patient still had high 
CSF output (> $200 \mathrm{ml} /$ day) after seven days, the EVD was clamped for 24 hours to challenge the drainage. During this period, strict GCS monitoring was performed. Any drop in GCS of two or more points was considered significant, and the EVD was then unclamped immediately. If the GCS remained the same, a repeat CT brain was done after 24 hours of clamping. If the repeat CT brain showed the presence or worsening of hydrocephalus, the patient was categorised as shunt dependent and required placement of a ventriculo-peritoneal shunt.

\section{Outcome and statistical analysis}

All patients were monitored for shunt dependency at two weeks and three months after the procedure. The difference in each patient's Graeb scores was calculated by comparing the CT brain done on arrival with another scan done within 24 hours after the surgical procedure. The score reductions were categorised as 1-2 points, 3-4 points, and more than 5 points. Patients in both groups underwent weaning from their EVDs over a period of days, as required. The appropriate EVD height was determined by the drainage amount, and the length of the weaning period was categorised into 1-2 days, 3-6 days, and more than 7 days. The final functional outcome was assessed at six months after the procedure. Data entry and analysis were done using an IBM SPSS Statistics 21. Pearsons' chiSquare test was used to determine significant differences in outcomes based on shunt dependency, Graeb scores, the durations needed for weaning from external ventricular catheters, and clinical outcomes. STATA software version 8.o was used to calculate the $P$-value for Fisher's exact test. Variables with $P$-values less than 0.05 were considered to be statistically significant.

\section{Results}

\section{Demographic data}

From January 2010 to January 2014, 39 patients with massive intraventricular haemorrhages were treated at our center; of these, 16 were managed with endoscopic washout and 23 with external ventricular drainage. Their ages ranged from 10-80 years, with a mean age of 51.3 years $(S D=15.8)$. There were 24 male and 15 female patients. Nine of the patients did not have any previous comorbidities; 21 patients had one comorbidity, with hypertension being the most common pathology, followed by diabetes mellitus. The pre- and post-operative CT brain scans of one of our patients with hypertensive IVH are shown below (Figure 1). The remaining nine patients had two or more comorbidities. On admission, the patients' clinical condition was evaluated using the Glasgow Coma Scale. Seven patients (17.9\%) presented with a GCS of $13^{-15}$, and the remaining 32 patients $(82.1 \%)$ had an initial GCS of $5-12$. This demographic data is tabulated in Table 2. CT brain scans done pre and post operatively to assess the severity of the intraventricular haemorrhage and its grading based on the Graeb scoring system are shown in Table 1.

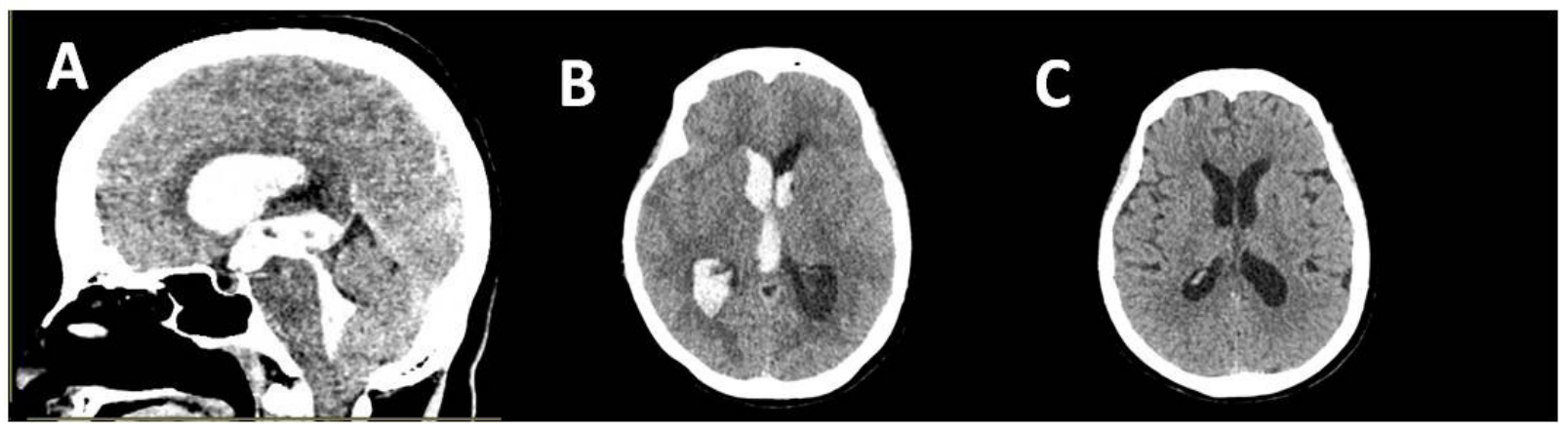

Figure 1. A and B: Preoperative images of massive intraventricular haemorrhage. C: CT brain after 24 hours of the surgery 
Original Article | Endoscopy in massive intraventricular haemorrhage

Table 1. Graeb grading system for intraventricular haemorrhage (Graeb et al., 1982)

\begin{tabular}{ccccc} 
Location of clot & Trace of blood & Less than $\mathbf{5 0 \%}$ filled & More than $\mathbf{5 0 \%}$ filled & $\begin{array}{c}\text { Completely filled and } \\
\text { expanded }\end{array}$ \\
\hline Lateral ventricles & 1 & 2 & 3 & 4 \\
Location of clot & $\begin{array}{c}\text { No blood } \\
\text { present }\end{array}$ & $\begin{array}{c}\text { Blood present, } \\
\text { ventricle normal size }\end{array}$ & $\begin{array}{c}\text { Filled with blood and } \\
\text { expanded }\end{array}$ & - \\
3rd ventricle & 0 & 1 & 2 & - \\
4th ventricle & 0 & 1 & 2 & - \\
\hline
\end{tabular}

* The total score is the addition of both lateral ventricles score, 3 rd ventricle score and 4th ventricle score (Score range 2-12)

Table 2. Demographic and clinical data for patients with IVH

\begin{tabular}{|c|c|c|c|}
\hline & $\begin{array}{c}\text { Endoscopic washout } \\
n(\%)\end{array}$ & $\begin{array}{l}\text { External ventricular } \\
\text { Drainage } n(\%)\end{array}$ & $P$ value \\
\hline \multicolumn{4}{|l|}{ Age (years) } \\
\hline $10-19$ & $2(100)$ & $\mathrm{o}(0.0)$ & $0.582^{b}$ \\
\hline $20-49$ & $7(50)$ & $7(50)$ & \\
\hline $50-69$ & $3(15.8)$ & $16(84.2)$ & \\
\hline$>70$ & $4(100)$ & $\mathrm{o}(0.0)$ & \\
\hline \multicolumn{4}{|l|}{ Gender } \\
\hline Male & $9(37.5)$ & $15(62.5)$ & $0.571^{\mathrm{a}}$ \\
\hline Female & $7(46.7)$ & $8(53 \cdot 3)$ & \\
\hline Ethnicity & & & $0.233^{\mathrm{b}}$ \\
\hline Malay & $12(37.5)$ & $20(62.5)$ & \\
\hline Chinese & $3(50)$ & $3(50)$ & \\
\hline Indian & $1(100)$ & $\mathrm{o}(0.0)$ & \\
\hline \multicolumn{4}{|l|}{ Others } \\
\hline \multicolumn{4}{|l|}{ GCS Score on admission } \\
\hline GCS $13-15$ & $4(57.1)$ & $3(42.9)$ & $0.509^{b}$ \\
\hline GCS 5-12 & $12(37.5)$ & $20(62.5)$ & \\
\hline $\mathrm{GCS}_{3}-4$ & $\mathrm{o}(0.0)$ & $\mathrm{o}(0.0)$ & \\
\hline \multicolumn{4}{|l|}{ Mean (SD) } \\
\hline Etiology & & & $0.153^{\mathrm{b}}$ \\
\hline Thalamic ICB & $3(25)$ & $9(75)$ & \\
\hline Basal Ganglia ICB & $7(43.8)$ & $9(56.2)$ & \\
\hline Ruptured AVM & $3(100)$ & $\mathrm{o}(0.0)$ & \\
\hline Tumoural & $1(50)$ & $1(50)$ & \\
\hline Pure IVH & $1(20)$ & $4(80)$ & \\
\hline Traumatic IVH & $1(100)$ & $\mathrm{o}(0.0)$ & \\
\hline Graeb score on admission & & & $0.473^{\mathrm{a}}$ \\
\hline$\leq 6$ & $1(25)$ & $3(75)$ & \\
\hline$>6$ & $15(42.9)$ & $20(57.1)$ & \\
\hline
\end{tabular}

a Pearson's chi-square assumptions met

${ }^{\mathrm{b}}$ Fisher's exact test, by STATA software 


\section{Shunt dependency and clinical outcome}

The drainage dependency at two weeks and three months post procedure was much lower in patients treated with an endoscopic washout. At two weeks, the rate of drainage dependency in the endoscopic washout group was $12.5 \%$ as compared to $56.5 \%$ in the external ventricular drainage group $(P=0.005)$ (Table 3$)$. At three months, the drainage dependency was $18.8 \%$ in patients treated with an endoscopic washout as compared to $65.2 \%$ in patients treated with an external ventricular drainage $(P=0.004)$ (Table 3 ). There was a significant reduction in Graeb scores after an endoscopic washout as compared to an external ventricular drainage $(P=0.001)$. More than $50 \%$ of patients who underwent an endoscopic washout had a Graeb score reduction of more than 5 points. In contrast, none of the patients in the external ventricular drainage group had a score reduction of more than 5 points. Most of the patients in the external ventricular drainage group (15 patients, 65.2\%) demonstrated a reduction of only 1-2 points (Table 3). None of the patients who underwent an endoscopic washout developed rebleeding.

In reference to the number of days needed to wean off an EVD in the postoperative period, a significant difference was noted between the endoscopic washout group and the external ventricular drainage group $(P=0.007)$. Most of the patients who underwent an endoscopic washout (81.2\%) required an EVD for 3-6 days. None of the patients in the endoscopic washout group required an EVD for more than seven days. In the external ventricular drainage group, six of the patients $(26.1 \%)$ needed an EVD for more than seven days, and 17 of them (73.9\%) required an EVD for 3-6 days. None of the patients in the external ventricular drainage group could be weaned from an EVD in 1-2 days, as opposed to the endoscopic washout group in which three patients (18.8\%) needed an EVD for only 1-2 days (Table 3). Functional outcomes were measured six months after the procedure using a modified Rankin scale score. Although there was no statistically significant difference in the functional outcomes of both groups with regard to the treatment instituted $(P=0.195)$, a considerably better outcome (mRs $0-4$ ) was observed in the endoscopic washout group (68.8\%) as compared to the external ventricular drainage group (48.7\%) (Table 3).

Table 3. Results for patients with IVH

\begin{tabular}{|c|c|c|c|}
\hline & $\begin{array}{l}\text { Endoscopic Washout } \\
\qquad(n=16)\end{array}$ & $\begin{array}{l}\text { External Ventricular Drainage } \\
\qquad(n=23)\end{array}$ & $P$-value \\
\hline \multicolumn{4}{|l|}{ Shunt dependency } \\
\hline at 2 weeks & $2(12.5)$ & $13(56.5)$ & $0.005^{\mathrm{a}}$ \\
\hline at 3 months & $3(18.8)$ & $15(65.2)$ & $0.004^{\mathrm{a}}$ \\
\hline \multicolumn{4}{|l|}{ Graeb score reduction } \\
\hline $1-2$ points & $1(6.2)$ & $15(65.2)$ & $0.001^{\mathrm{a}}$ \\
\hline $3-4$ points & $6(37.5)$ & $8(34.8)$ & \\
\hline$>=5$ points & $9(56.2)$ & o (o.o) & \\
\hline \multicolumn{4}{|l|}{ EVD weaning days } \\
\hline 1-2 days & $3(18.8)$ & o (o.o) & $0.007^{\mathrm{b}}$ \\
\hline $3-6$ days & $13(81.2)$ & $17(73.9)$ & \\
\hline$>=7$ days & o (o.o) & $6(26.1)$ & \\
\hline \multicolumn{4}{|l|}{ mRs at 6 months } \\
\hline o-4 (good outcome) & $11(68.8)$ & $11(48.7)$ & $0.195^{\mathrm{a}}$ \\
\hline 5-6 (poor outcome) & $5(31.2)$ & $12(52.2)$ & \\
\hline
\end{tabular}

a Pearson's chi-square assumptions met

${ }^{\mathrm{b}}$ Fisher's exact test, by STATA software 
Original Article | Endoscopy in massive intraventricular haemorrhage

\section{Discussion}

\section{Pathomechanics of IVH}

Massive intraventricular haemorrhage is a devastating neurosurgical condition which occurs commonly in patients with an intracerebral haemorrhage (ICH). This condition carries a high mortality rate of $42.6 \%-83.3 \%$ (3). A thalamic $\mathrm{ICH}$ is seen in $10 \%-15 \%$ of all the cases of intracerebral haemorrhage as reported by Chen et al. (4), and it commonly extends into the ventricular system. Blood clots within the ventricular system lead to acute obstruction of the cerebrospinal fluid and a sudden rise in intracranial pressure as the compensatory mechanism is compromised. The presence of clots deep within the cortical or ventricular structures is also related to a reduced level of consciousness as the function of the ascending reticular activating fiber system (ARAS) is diminished, rendering the patient ventilator dependent and requiring prolonged intensive care unit (ICU) admission. As the clot within the ventricular system undergoes lysis, the fibrin degradation products (FDPs) and bilirubin oxidation products (BOx) that are formed during the breakdown process will enter and circulate in the cerebrospinal fluid. FDPs and BOx will then reach the arachnoid granulation and cause delayed communicating hydrocephalus.

\section{Management options for IVH}

The current standard management of massive intraventricular haemorrhage is placement of an external ventricular drainage catheter. However, this procedure addresses only the sequelae of hydrocephalus and increased intracranial pressure. In order to address all three devastating clinical sequelae caused by intraventricular haemorrhage, various authors have studied the use of neuroendoscopic surgery $(4-7)$. The purpose of this study was to explore a method of managing massive intraventricular haemorrhage which is minimally invasive and at the same time provides the maximal clinical benefit. Endoscopic washout is the only management option that fits these criteria.

\section{Group comparison in our study}

The results of this study showed less drainage dependency and greater reduction in Graeb score in the endoscopic washout group as compared to the EVD-treated group. Significantly fewer days were needed to wean from an EVD in the endoscopic group. The reasons for this positive clinical outcome may be: (i) rapid removal of the hematoma which alleviated the potential neurotoxic effect of blood clots; (ii) rapid relief of obstructive hydrocephalus which lowered the ICP and improved the cerebral perfusion pressure (CPP); (iii) direct visualisation of the clot and almost complete removal of the hematoma which contributed to greater reduction in the Graeb score; (iv) catheter placement done under direct vision which lowered the possibility of obstruction by choroid plexus; (v) evacuation of the intraventricular hematoma which reduced the chances of catheter blockage by thick clots and poor flow; (vi) rapid re-establishment of CSF flow via the natural conduits which led to a shorter duration needed to wean from an EVD.

\section{Functional outcome at six months post- procedure}

This study revealed that the application of neuroendoscopy for massive intraventricular haemorrhages did not alter the functional outcome for the patients at six months. This is because the functional outcome is mainly determined by the initial GCS on arrival and the primary pathology that caused the intraventricular haemorrhage $(8,9)$. However, there was considerable difference in the good outcome (mRs $0-4)$ demonstrated in the endoscopic washout group (68.8\%) as compared to the external ventricular drainage group (48.7\%). This difference did not yield statistical significance as the sample size was small, and hence is a limitation of this study.

\section{Conclusions}

The use of neuroendoscopy in patients with a massive IVH has significantly reduced drainage dependency and produced good clinical outcomes. This may be related to the early removal of hematomas, the creation of new cerebrospinal diversion pathways, and the dilution of the fibrin degradation products (FDPs) and bilirubin oxidation products (BOx) which can damage the arachnoid granulations. This study also revealed a potentially better functional outcome through neuroendoscopy with a considerable advantage in the modified Rankin scale score. The lack of statistically significant results could be due to the small sample size. 


\section{Acknowledgement}

All authors certify that they have no affiliations with or involvement in any organisation or entity with any financial interest or non-financial interest in the subject matter or materials discussed in this manuscript.

\section{Authors' Contributions}

Conception and design: JR, ZI, JMA, AA, SH

Analysis and interpretation of the data: JR, ZI, JMA, AA, SH

Drafting of the article: JR, ZI, JMA, AA, SH

Critical revision of the article for important intellectual content: JR, ZI, JMA, AA, SH

Final approval of the article: JR, ZI, JMA, AA, SH Provision of study materials or patients: JR, ZI, JMA, AA, SH

Statistical expertise: JR, ZI, JMA, AA, SH

Collection and assembly of data: JR

\section{Correspondence}

Dr Jason Raj Johnson

MBBS (Malaya), Master of Surgery (Neurosurgery) USM

Department of Neurosurgery,

Kuala Lumpur General Hospital,

Jalan Pahang,

50586 Kuala Lumpur,

Malaysia

Tel: +6097676299

Fax: +6 o9 7648613

E-mail: jasonrj81@gmail.com

\section{References}

1. Hanley DF, Naff NJ, Harris DM. Intraventricular Hemorrhage: Presentation and Management Options. Seminars in Cerebrovascular Diseases and Stroke. 2005;5(3):209-216. https://dx.doi. org/10.1053/j.scds.2006.01.011

2. Yadav YR, Mukerji G, Shenoy R, Basoor A, Jain G, Nelson A. Endoscopic management of hypertensive intraventricular haemorrhage with obstructive hydrocephalus. BMC Neurol. 2007;7:1-9. https://dx.doi.org/10.1186/14712377-7-1

3. Adams RE, Diringer MN. Response to external ventricular drainage in spontaneous intracerebral hemorrhage with hydrocephalus. Neurology.
1998;50(2):519-523. https://dx.doi.org/10.1212/ WNL.50.2.519

4. Chen CC, Liu CL, Tung YN, Lee HC, Chuang $\mathrm{HC}$, Lin SZ, et al. Endoscopic surgery for intraventricular hemorrhage (IVH) caused by thalamic hemorrhage: comparisons of endoscopic surgery and external ventricular drainage (EVD) surgery. World Neurosurg. 2011;75(2):264-268. https://dx.doi.org/10.1016/j.wneu.2010.07.041

5. Zhang Z, Li X, Liu Y, Shao Y, Xu S, Yang Y. Application of neuroendoscopy in the treatment of intraventricular hemorrhage. Cerebrovasc. 2007;24(1):91-96. https://dx.doi. org/10.1159/000103122

6. Basaldella L, Marton E, Fiorindi A, Scarpa B, Badreddine H, Longatti P. External ventricular drainage alone versus endoscopic surgery for severe intraventricular hemorrhage: a comparative retrospective analysis on outcome and shunt dependency. Neurosurg Focus. 2012;32(4):E4. https://dx.doi. org/10.3171/2012.1.FOCUS11349

7. Idris Z, Raj J, Abdullah JM. Early experience in endoscopic management of massive intraventricular hemorrhage with literature review. Asian J Neurosurg. 2014;9:124-129. https://dx.doi.org/10.4103/1793-5482.142731

8. Diringer MN, Edwards DF, Zazulia AR. Hydrocephalus: a previously unrecognized predictor of poor outcome from supratentorial intracerebral hemorrhage. Stroke. 1998;29(7):1352-1357. https://dx.doi. org/10.1161/01.STR.29.7.1352

9. Longatti P, Fiorindi A, Martinuzzi A. Neuroendoscopic aspiration of hematocephalus totalis: technical note. Neurosurgery. 2005;57(Suppl 4):E409. https://dx.doiorg/10. 1227/o1.neu.0000176702.26810.b7

10. Graeb DA, Robertson WD, Lapointe JS, Nugent RA, Harrison PB. Computed tomographic diagnosis of intraventricular hemorrhage. Etiology and prognosis. Radiology. 1982;143(1):91-96. https://dx.doi.org/10.1148/ radiology.143.1.6977795

11. LeRoux PD, Haglund MM, Newell DW, Grady MS, Winn HR. Intraventricular hemorrhage in blunt head trauma: an analysis of 43 cases. Neurosurgery. 1992;31(4):678-684. https:// dx.doi.org/10.1227/00006123-199210000-00010 